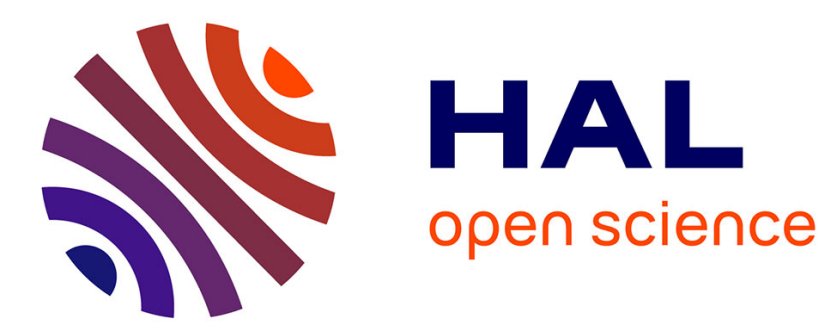

\title{
L'Ennemie dans l'Europe en guerre au XXe siècle
}

\author{
Fabrice Virgili
}

\section{To cite this version:}

Fabrice Virgili. L'Ennemie dans l'Europe en guerre au XXe siècle. L'Autre, 2002, Dossier L'ennemi, 3 (1), pp.39-51. halshs-01360445

\section{HAL Id: halshs-01360445 https://shs.hal.science/halshs-01360445}

Submitted on 29 Nov 2018

HAL is a multi-disciplinary open access archive for the deposit and dissemination of scientific research documents, whether they are published or not. The documents may come from teaching and research institutions in France or abroad, or from public or private research centers.
L'archive ouverte pluridisciplinaire HAL, est destinée au dépôt et à la diffusion de documents scientifiques de niveau recherche, publiés ou non, émanant des établissements d'enseignement et de recherche français ou étrangers, des laboratoires publics ou privés. 
Fabrice Virgili « L'Ennemie dans l'Europe en guerre au XXe siècle ", L'Autre. Cliniques, cultures et sociétés, 2002, vol.3, n¹, p. 39-51.

Désigné, combattu, blessé, fait prisonnier ou tué lors de l'affrontement guerrier, l'ennemi est certes une figure extrême de l'altérité, il n'en demeure pas moins un homme que l'on combat en tant qu'homme. Dans le champ des assignations traditionnelles entre les sexes, la guerre est le domaine des hommes. Elle devient même au cours du XIXème siècle le domaine de tous les hommes, avec, en France à partir de la Révolution, une armée nationale et des soldats citoyens. Le sacrifice de soi pour la patrie, la geste combattante déclinée sous ses formes les plus hérö̈ques, deviennent constitutifs d'une identité masculine et virile renouvelée au cours du siècle (Mosse, 1997).

En août 1914, ce sont des hommes, français, qui s'apprêtent à " mourir pour la patrie ", et partent combattre, tuer, d'autres hommes, allemands, prêts eux aussi à "mourir pour l'empereur et la patrie". Pourtant, alors que se déroulent ces corps à corps masculins et meurtriers, les femmes ainsi que diverses figures du féminin sont également mobilisées. Bien entendu, dans et pour son camp, mais aussi, dans celui de l'adversaire : l'ennemi est-il aussi une ennemie?

Il ne s'agit pas ici de brosser une histoire des femmes dans la guerre, mais de voir comment les guerres modernes, et l'on pense en premier lieu aux deux conflits mondiaux, en ce qu'elles mobilisent la société tout entière font également de l'ennemi, des femmes, et des femmes, des ennemis.

\section{Dire l'ennemi}

Si l'on accepte l'idée que la construction de la figure de l'ennemi repose sur des images fondamentales de l'altérité et qu'elle participe tout autant à l'image de soi, il faut s'interroger sur le sens que peut avoir la féminisation de l'ennemi. Non pas la place des femmes dans le camp adverse, sur laquelle nous reviendrons, mais la place de certaines figures du féminin utilisées comme mode de désignation de l'autre belligérant.

Les discours de mobilisation s'appuient autant sur l'image de son camp, défenseur de la civilisation, que sur celle du camp adverse, celui de la barbarie. Déclinés, selon les conflits ou les groupes qui l'énoncent en autant d'antagonismes absolus, qu'ils soient d'ordres nationaux, idéologiques, ou ethniques, l'opposition parce qu'elle accompagne l'affrontement, tend à rejeter l'ennemi dans l'inhumain.

Cependant, le processus n'est en rien homogène ni linéaire et les registres de discours mobilisés sont forts variés. L'ennemi peut être assimilé à une brute, souvent sanguinaire, par un discours de criminalisation : c'est la figure archaïque et revisitée du barbare. La violence est pour lui un plaisir qui le pousse à enfreindre "par nature" les règles. Celles de sa communauté, mais étendues à l'humanité tout entière. Au cours du premier conflit mondial, les mains coupées des enfants belges ou des départements du nord de la France, sont une rumeur récurrente qui alimente tout au long de la guerre l'image de la barbarie allemande (Horne et Kramer, 2001). À l'inverse, la présence de troupes coloniales noires dans l'armée française et les rumeurs qui s'y attachent (colliers d'oreilles ou de doigts coupées à l'adversaire) est, aux yeux des Allemands, la preuve tangible de la barbarie française.

Une seconde forme de désignation est la métaphore animale. Là aussi les formes sont infinies. Qu'il s'agisse de prédateurs (loup, tigre), qui renvoient à la sauvagerie de l'autre autant qu'au risque pour soi, d'animaux d'élevage (porcs, bœufs ou moutons) qui se nourrissent sur le 
pays ou suivent aveuglément leurs chefs, ou encore de tout un univers d'insectes (puces, poux, doryphores), dont le caractère innombrable, la voracité, la saleté dont ils sont les symptômes, sous-tendent la nécessité d'un véritable nettoyage. Ce dernier aspect renvoie à des discours prophylactiques que l'on retrouve dans différents contextes où l'ennemi ne revêt plus que les contours d'une maladie (cancer, infection, maladies vénériennes). Ce mode de désignation en soulignant la nécessité de la purification, qu'elle soit politique, raciale ou nationale, place de fait l'ennemi au sein de la communauté. C'est la nation française qu'il faut purifier à la libération "des miasmes de l'infection bochisante" .

Ces modes de désignation ne sont pas propres aux temps de guerre. Ils trouvent leur source dans les imaginaires mentaux de chaque groupe, ils puisent dans le registre souvent familier de l'insulte, du rejet de l'autre. Traiter quelqu'un de chien, de pouilleux, d'infection ne révèle dans la plupart des cas, ni le désir, ni la réalité de la mise à mort de l'autre. En ce sens, ils ne peuvent à eux seuls, être les témoins d'une volonté exterminatrice et doivent être considérés dans un ensemble de discours, de pratiques et de représentations de ce que l'on appelle communément la culture de guerre.

Cette liste ne prétend pas à l'exhaustivité et une réflexion plus approfondie sur les innombrables manières de dire l'ennemi mériterait d'être menée. N'ont pas été évoqués cidessus par exemple les nombreux détournements des symboles nationaux. Parmi eux, à nouveau des animaux, tels le coq français, l'aigle allemand ou le lion britannique pour prendre les plus connus, mais aussi des femmes : Marianne et Germania.

\section{La féminisation de l'ennemi.}

La France et l'Allemagne se sont respectivement dotés d'une figure féminine. Néanmoins dans les représentations diverses des relations entre les deux nations c'est bien l'image d'un couple qui domine. En 1934, Jules Romain dans l'avant propos de son ouvrage "Le couple France-Allemagne" justifiait le titre choisi en ces termes : "il y a bien dans l'histoire francoallemande, dans le drame séculaire de ces deux peuples, dans les attirances et les haines, également ardentes, qu'ils éprouvent d'âge en âge l'un pour l'autre, et surtout du côté allemand, quelque chose de sexuel, quelque chose qui semble une transposition du sexuel dans le collectif" (Romains, 1934, p.XI). Couple inscrit dans la géographie de l'Europe et dont la figure traverse le temps de paix comme celui de la guerre. Pourtant si Jules Romains n'assigne à aucune nation son rôle, il ne fait guère de doute que la France en est l'élément féminin. Cette répartition sexuée est encore plus manifeste en temps de guerre. Ainsi, Wolfgang Geiger souligne à quel point la victoire de juin 1940 est aux yeux des Allemands celle "de la virilité germanique sur une nation efféminée, barricadée derrière une ligne Maginot sans valeur" (Geiger, 1999, p.200). Construire alors une image de l'ennemi au féminin, c'est placer la relation à l'autre dans un rapport de domination " naturelle ". Si l'adversaire est féminin, il est logique qu'il soit vaincu et soumis. Par contre, en 1944, la libération de la France s'accompagne du côté français d'un discours de la virilité retrouvée. Celle-ci n'empêche pas de constater la prégnance des stéréotypes du féminin. Les entretiens, en août 1944, entre le commandant allemand von Choltitz et le consul général de Suède Raoul Nordling sont de ce point de vue évocateurs. Leurs discussions portent en premier lieu sur le sort des prisonniers politiques, la répression de l'insurrection, une trêve des combats de la libération, la destruction de Paris. Mais, à deux reprises, von Choltitz évoque Paris sous les contours du féminin. Il s'agit d'abord d'une jeune fille qui passe en vélo devant l'hôtel Meurice où se trouvent les deux hommes et dont le vent soulève la jupe ; von Choltitz sourit : "On n'a pas le droit de tirer sur elle et ses pareilles". Trois jours plus tard, il dit à propos la capitale ; "C'est comme une jolie femme, dit-il, quand elle vous donne une gifle, on ne la rend pas" (Nordling, 2002). Deux discussions au cours desquelles est évoqué le sort de Paris et où l'on retrouve une

\footnotetext{
${ }^{1}$ Union champenoise (L'), Reims, 1 ${ }^{\mathrm{er}}$ septembre 1944.
} 
représentation de la ville sous les contours d'un féminin, symbole de jeunesse, d'innocence et de beauté. On ne peut que relever le décalage entre une certaine image de la féminité et le vécu des femmes, qui loin de parcourir la guerre, insouciantes au danger, en ont traversé les épreuves au même titre que les hommes. L'évocation par un général allemand, qui a combattu auparavant sur le front de l'Est, de la présence de femmes qui interdirait tout combat, laisse perplexe. Quand on sait les conditions dans lesquelles s'est déroulée l'offensive contre l'URSS, le nombre de victimes civiles qu'elle a engendré (bombardements, représailles, groupe d'extermination) quel sens donner à cette remarque ? Nommé sur le front de l'Ouest, von Choltitz se retrouve dans un contexte où la brutalité générale du conflit est indéniablement un degré en dessous, mais où il accumule les retraites. L'évocation d'une image, certes stéréotypée, mais pacifiée du féminin ne révèle-t-elle pas une faille dans une culture de guerre, le retour d'un imaginaire déréalisé de la guerre ? Quoi qu'il en soit, la description d'un ennemi au féminin place bien l'adversaire hors du cadre d'un affrontement viril et loyal. Tout tend lui dénier sa légitimité à se trouver sur le champ de bataille.

Dans le discours de guerre, la féminisation de l'ennemi passe également par une mise en cause de sa virilité. S'il y a homosexualité, elle est toujours celle de l'autre, d'un ennemi qui, par ses pratiques sexuelles, s'exclut de la masculinité, n'est pas digne d'un affrontement "entre hommes ". Pendant la Grande guerre, Louise Thuliez condamnée à mort par les Allemands pour avoir favorisé l'évasion de nombreux soldats belges français et britanniques, puis graciée, est l'une des rares femmes décorée de la Croix de guerre. En avril 1919 dans la Revue des deux mondes, elle fait le récit de "sa guerre". Celle-ci commence en août 1914 quand les Allemands arrivent dans le village du nord où elle se trouvait alors en vacances: "Là où ils [les soldats Allemands] trouvaient un piano, ils s'y installaient, chantaient et dansaient. Revêtus des vêtements féminins qu'ils avaient volés. Et je ne puis exprimer le dégoût éprouvé à voir des soldats à cheval, affublés de robes de femmes qu'ils abritaient sous des ombrelles blanches. C'étaient bien les barbares dont on nous avait parlé " (Thuliez, 1919, p. 652). Participant au rejet des ennemis de la nation, la condamnation du franchissement de la barrière du genre est autant partagé par les hommes que par les femmes.

Lors de l'occupation de la Ruhr par les Français de 1919 à 1930, les journaux nationalistes allemands accusent les autorités françaises d'avoir installé des lieux de prostitution dans les différentes villes sous leur contrôle. Parmi les rumeurs qui circulent alors celle de bordels d'hommes réservés aux soldats homosexuels de l'armée française. Dans un courrier adressé au Ministre des Affaires étrangères, Aristide Briand, le Haut Commissaire de la République française dans les Provinces du Rhin réfute l'accusation: "l'allégation relative aux maisons de prostitution masculine est un audacieux mensonge". Il poursuit: "Le vice contre nature est très répandu en Allemagne, maintenant comme avant la guerre. C'est un fait de notoriété publique, et ce vice est même qualifié de "vice allemand". Si des établissements de ce genre existent en Allemagne, ce sont des établissements purement allemands. Non seulement les troupes d'occupation les ignorent et entendent les ignorer, mais la " débauche allemande " est un des faits qui répugne le plus aux Français et qui contribue à maintenir un fossé moral entre les populations des deux pays."

Le travestissement, l'homosexualité, la débauche rejettent l'ennemi dans une altérité sexuelle et morale. Ils ne sont pas renvoyés à l'autre sexe biologique, mais leurs pratiques perçues comme barbares ou contre-nature, en les excluant du masculin, les placent dans un entre-deux insupportable, car du point de vue du stéréotype, la frontière du genre se doit d'être tranchante.

\footnotetext{
${ }^{2}$ Lettre du 5 octobre 1927, Archives nationales AJ-9-3216.
} 


\section{La femme de l'ennemi}

Que l'on retrouve ou non des formes de féminisation du pays ennemi ou de ses combattants, cela n'implique pas forcément la prise en compte des femmes du camp adverse. La perméabilité croissante entre civil et militaire au cours du XXe siècle, l'élargissement du champ de bataille à la société tout entière, ont inclus de plus en plus largement les femmes dans la guerre. Pourtant, avant d'être une ennemie, elles sont d'abord la femme de l'ennemi.

\section{Des femmes invisibles}

Dans un face à face guerrier exclusivement masculin, la femme de l'ennemi n'est guère présente. Que l'adversaire ait une fiancée, une épouse ou une mère en fait au contraire un camarade de misère. L'éloignement de son foyer dont peuvent témoigner les photographies qu'il porte sur lui renvoi au sort commun des combattants. En restituant la filiation : ils ont aussi une mère; en laissant entrevoir la possible tendresse de l'autre, la femme de l'ennemi redonne à ce dernier une certaine humanité.

Il en est autrement quand les populations civiles deviennent la cible des combattants. Femmes, enfants et vieillards sont tués, en infraction des lois de la guerre mais surtout de l'imaginaire mental des sociétés en question. Comment faire accepter le massacre des civils adverses alors que la mise à mort de "nos fils et nos compagnes" est bien souvent au cœur de la dénonciation de la barbarie ennemie ? Ce n'est pas ici, l'universel masculin qui tait la souffrance des femmes de l'autre camp, mais bien une vision sexuée du monde. Les femmes ne sont pas oubliées parce qu'invisibles socialement aux yeux des militaires, elles sont tues car leur mise à mort explicite enfreint les lois de la guerre. Elles y représentent la faiblesse, comme les enfants l'innocence. L'ennemi ne peut dans ces circonstances être décliné entre hommes, femmes et enfants, seule sa vision comme un tout rend possible la mise à mort de chacun de ses membres quel que soit son sexe ou son âge. Lee Miller, photographe de guerre accréditée par l'armée américaine $e^{3}$ a multiplié, de Normandie en Allemagne, les portraits resserrés. Pourtant, derrière l'objectif, les visages, les individus qu'elle photographie dans l'Allemagne en ruine ne se dissocient pas d'une vision globale de l'adversaire: "Les enfants jouent avec des échasses, des billes des toupies, des cerceaux (...) les mères cousent, balaient, cuisinent et les paysans labourent hersent, tout comme des gens réels. Mais ils sont l'ennemi "

Le bombardement stratégique, c'est-à-dire la destruction massive de population civile afin d'affaiblir le moral du pays ennemi au point d'amener son gouvernement à la capitulation, pose des problèmes du même ordre. Sous le déluge de bombes, hommes et femmes sont égaux devant la mort. Les deux sexes partagent une expérience de guerre; ils ont alors, contrairement au front, un sort commun de victimes (Voldman, 1997). Mais du côté des bombardiers, le choix d'une mise à mort massive de civils nécessite une distanciation plus grande vis-à-vis d'un adversaire non combattant. L'altitude, les raids nocturnes y contribuent mais c'est surtout dans la désignation de celui-ci que la déréalisation est la plus forte. L'objectif est alors un nom de ville; atteint il devient une photographie aérienne et un chiffre : celui des victimes. Les frontières d'âge et de genre doivent s'effacer, l'ennemi n'est ni masculin ni féminin il est un point du territoire désormais détruit. Quels que soient les arguments d'ordre militaire qui justifient le bombardement (dommages collatéraux, objectif stratégique), les victimes surtout si elles incarnent la vulnérabilité et l'innocence se doivent d'être invisibles.

\section{L'ennemie, objet de conquête violente et virile}

Dans une situation de conquête ou d'occupation, les femmes du territoire envahi doivent comme les hommes se soumettre au nouveau maitre. Le contrôle des populations, les

\footnotetext{
3 Au cours de la Seconde Guerre mondiale, 117 femmes ont été accréditées par l'armée américaine en tant que journalistes de presse ou de radio, photographes.

${ }^{4}$ Miller L., "Dans l'Allemagne vaincue” Vogue, juin 1945. Cité par AMAr M., "Quelques mois dans la vie de Lee Miller" Voir ne pas voir la guerre, BDIC-Somogy, Paris, 2001, p. 126-127.
} 
déplacements forcés, la répression de toute manifestation d'hostilité, en une formule " la loi du vainqueur " s'applique à tous. Cela n'exclut pas, bien au contraire un comportement spécifique de l'homme conquérant vis-à-vis des femmes du pays vaincu. Par la force, ou dans le cadre plus complexe de rapports de séduction, les femmes de l'ennemi doivent également d'être conquises.

Le "viol de guerre" est présent dans bien des récits de conquête. L'image d'une soldatesque assouvissant ses pulsions viriles, s'emparant de ce " butin féminin " est récurrente dans les récits des atrocités ennemies. Mais si le traumatisme est probablement le même pour toutes celles qui en sont victimes, l'expression uniforme de "viol de guerre" mérite d'être questionnée. Il serait plus juste de parler de viols en temps de guerre afin de mieux distinguer les différences de contexte, de pratique et de sens.

Les viols commis en temps de guerre ont en commun le sentiment d'impunité partagé par leurs auteurs et le contexte de violence extrême propre à la période. Violence du champ de bataille, de soldats qui côtoient quotidiennement la mort ; violence envers les civils, dont les bombardements, les pillages et les massacres ne sont qu'une trop brève évocation. On relève dans cette situation une criminalité sexuelle en temps de guerre. Les victimes peuvent aussi bien être des femmes ennemies, à propos desquelles le sentiment d'impunité est le plus fort, mais aussi des compatriotes ou des alliées. C'est par exemple le cas des viols commis par des soldats américains en France en 1944 et 1945. Manifestement, la répression des crimes sexuels commis par la troupe n'est pas une priorité des autorités militaire. Un désintérêt, voir une indulgence qu'on ne saurait pourtant assimiler à un encouragement. Le viol reste un crime aux yeux de la plupart des codes militaires et quand le cas est trop flagrant ou l'accusation persistante, les soldats sont poursuivis et condamnés, parfois à mort, par les tribunaux militaires.

Les victimes sont des femmes de tout âge dont l'appartenance au camp adverse n'est que secondaire. Les viols sont le plus souvent commis par des soldats qui échappent momentanément à l'encadrement de leur unité, il ne s'agit aucune ment dans ces cas de pratiques prémédités ou planifiées.

Il en est autrement des viols de conquête. Ceux-ci, étudiés par Stéphane Audoin-Rouzeau pour la Première Guerre mondiale (Audoin-Rouzeau, 1995), et plus récemment par Raphaëlle Branche pour la guerre d'Algérie (Branche, 2001), participent de la soumission de la nation ennemie. Les femmes de l'ennemi sont alors transformées en terrain à conquérir. L'impunité propre au temps de la guerre tend vers une légitimité, celle du vainqueur. Cruauté, humiliation, le viol participe à la soumission de l'adversaire. En violant les femmes de l'ennemi, c'est la société dans son ensemble qui se trouve également atteinte. Les hommes de n'avoir pu protéger "leurs" femmes; celles-ci par la souffrance, la honte qu'on leur a fait subir. Elles sont en premier lieu des victimes, mais aussi celles qui portent en elles le ferment de l'ennemi, une rupture dans la filiation qui menace toute la communauté. Comme l'écrit Raphaëlle Branche à propos de l'Algérie: “ À travers la femme, bousculée, violentée, violée, le soldat atteint sa famille, son village, et tous les cercles auxquels elle appartient jusqu'au dernier; le peuple algérien" (Branche, 2001, p.297).

On voit bien dans le cas de ces viols de conquête que les victimes ne le sont pas seulement en tant que femme, ni même femme de l'ennemi, mais bien femme de la nation ennemie. Le cas est encore plus marqué à propos des viols commis par des soldats de l'Armée rouge en Allemagne en 1945. Le phénomène a un caractère massif sans commune mesure avec les exemples cités jusque-là. Certaines estimations parlent de 200000 viols pour la seule région de Berlin (Naimark, 1995) et ce sans qu'ils soient planifiés. La dimension conquérante est ici démultipliée par le désir de revanche des soldats soviétiques. Il s'agit de faire payer à tous les Allemands et toutes les Allemandes l'effroyable somme de souffrance endurée depuis juin 1941. Plus qu'une impunité on peut y voir un véritable affaissement des barrières morales face à un ennemi, masculin ou féminin, tant haï. 
Dernier cas de figure, celui des viols systématiques observés plus récemment lors de la guerre en Bosnie. Contrairement aux situations précédentes, ces viols se sont déroulés de manière préméditée et systématique : c'est le cas des viols subis par les femmes bosniaques détenues dans le camps. L'objectif avoué était "de féconder ces femmes [bosniaques] afin qu'elles accouchent d'un petit serbe" et tout était fait pour s'assurer que la grossesse parviendrait à son terme. Le dessin des violeurs n'est pas seulement d'instaurer une domination immédiate par la terreur et l'humiliation mais de remplacer en tant que géniteur les ennemis qu'il a tué, d'utiliser les femmes de l'ennemi comme vecteur de leur propre destruction. Comme l'écrit Véronique Nahoum Grappe : "paradoxalement, le viol, qui veut posséder l'avenir de l'autre en germe au moment de la fécondation, tente aussi l'effacement de son passé, puisque ce germe du futur contient les "racines" identitaires du groupe : le viol veut refaire l'autre, le recommencer à son origine, dans le ventre de sa mère " (Nahoum-Grappe, 1997, p.177.)

Les femmes de l'ennemi, terrain de conquête virile, sont aussi les mères de l'ennemi. Matrices elles sont à détruire, non par la mise à mort, mais par le viol. Seul le sperme, dans un imaginaire sexué et asymétrique du masculin et du féminin, transmet le sang du vainqueur, assure la reproduction et le caractère définitif de sa domination.

\section{Séduire l'ennemie}

Lors de l'occupation de la France en juin 1940, les relations sexuelles prévisibles entre soldats allemands et femmes françaises devait, aux yeux de l'état major de la Wehrmacht, être exclusivement limitées aux prostituées encartées et sous contrôle des autorités d'occupation. Que les raisons en aient été, pour l'armée, la peur du péril vénérien, ou pour les SS celle d'une souillure raciale, la consigne a été progressivement abandonnée devant le nombre significatif de liaisons établies entre soldats occupants et Françaises. Selon Wolfgang Geiger, le regard allemand porté sur la femme française est des plus significatif du fossé culturel entrevu lors du quotidien de l'occupation. Le maquillage, le soucis de l'apparence des Françaises, renvoient à l'image d'une France superficielle. Représentation fortement prégnante, elle s'oppose au caractère "naturel" de la nation allemande et de ses femmes. La description de Françaises trop fardées, s’inscrit dans la représentation plus générale de la France pays ennemi : "Nous parlions avec des fantassins allemands qui s'étaient échappés de leur captivité en France. Ils nous rapportèrent, plein de dédain que des femmes fardées et attifées avaient percé les rangs des soldats de garde, seulement pour cracher à la figure des boches " (Geiger, 1999). Une fois le pays soumis, les ennemies devenues vaincues renvoient une image bien plus "fortement érotisée ". Tout au long de l'occupation, l'image de la Française et plus encore de la Parisienne, a conservé un attrait particulier pour les soldats de la Wehrmacht. La manière dont les Allemands perçoivent les femmes d'une nation ennemie qu'ils viennent de conquérir mérite de s'y arrêter.

Du côté français, les témoignages ne manquent pas qui décrivent, au moins au cours des premiers mois de l'occupation, des Allemands, disciplinés, corrects, galants, ou tout simplement grands et beaux. Par ailleurs, les soldats disposaient en France d'un pouvoir d'achat conséquent qui leur permettait de multiplier cadeaux et avantages en ces temps de pénurie. De fait, des relations se multiplièrent dès les premiers jours de l'occupation entre ces soldats et des Françaises. Il n'est pas aisé de distinguer ce qui dans ces relations est du domaine du sentiment, du désir, d'une recherche de protection, d'une amélioration du quotidien. Quel qu'en soient les motifs, elles sont pour chacun un moyen de mettre la guerre entre parenthèse. Elles permettent un repli, même temporaire, sur la sphère privée. Nonobstant, ces relations consentantes n'échappent pas à un rapport d'occupant à occupé. Le vainqueur considère sa séduction d'autant plus normale que les Françaises, bien qu'appartenant à la nation ennemie, se sont trouvées abandonnées par leurs hommes. Incapables d'empêcher l'invasion du territoire, les Français ont failli à leur rôle de protection, 
de père et d'époux. Le nouveau maître, à la virilité intacte, est alors l'Allemand, les femmes de l'ennemie sont devenues ses femmes. L'expression peut paraitre outrée, mais c'est bien comme "femmes à boches" qu'elles sont perçues à la Libération. La nationalité de l'amant, en l'occurrence allemand, leur retirant leur qualité de Française. De ce point de vue leur indépendance est niée et leur sexualité les fait passer dans le camp adverse. Adultère à la nation, elles deviennent des ennemies, mais de la France désormais.

De l'autre côté du Rhin, dans les fermes où étaient affectés de nombreux prisonniers français, d'autres rencontres, mais avec les femmes du vainqueur cette fois, ont également eu lieu. Les témoignages des prisonniers sont souvent discrets à propos de ces liaisons au cœur de l'Allemagne. Ils font état de liaisons ; clandestine car, contrairement à la France, le risque était alors immédiat et considérable. Les prisonniers de guerre étaient passibles de la peine de mort, les femmes allemandes de la déportation. Cela n'empêcha pas que des relations durent jusqu'à la fin de la guerre avec le désir de les prolonger au delà. Quelques-uns sont revenus après guerre en France avec une compagne de l'autre camp. Or, être dans ce cas la compagne d'un ancien prisonnier de guerre français ne constituait ni une protection ni un passeport. Interceptées à la frontière, ces Allemandes indésirables étaient renvoyées dans leur pays ; arrivées à destination en France elles rencontrait l'hostilité de la famille du voisinage. Contrairement à l'amant, allemand et occupant, dont la nationalité s'étendait à sa maitresse, la compagne du prisonniers de guerre français demeurait l'Allemande donc l'ennemie. La dissymétrie est plus complexe qu'elle n'en a l'air. Seules les Françaises qui ont eu des relations avec l'ennemi sont inquiétées, les hommes restent seuls maitre de leur sexualité, qu'elle que soit la nationalité de leur partenaire. Cependant, est-ce parce que le prisonnier de guerre fait parti du camp des vaincus? Sa relation avec une femme de l'ennemi ne fait pas pour autant de cette dernière une alliée, encore moins une Française. Le contexte de l'occupation, la force de l'antagonisme ainsi créé fait de l'autre, quel que soit son sexe, un ennemi immuable.

\section{De véritables ennemies.}

Filles, sœurs, épouses, mères de l'ennemi, les femmes restent bien souvent perçues à travers le prisme des assignations traditionnelles de chaque sexe. Pourtant au cours du XXe siècle elles sont nombreuses à s'approcher de plus en plus du combat. D'abord dans des tâches dévolues aux femmes: infirmières, cantinières, secrétaires, mais aussi dans certains cas comme combattantes. La délimitation sexuée de l'univers combattant se fait moins intangible et certains ennemis sont alors bien des ennemies.

La présence de femmes sur le champ de bataille, ou plutôt la participation des femmes au combat tant le champ de bataille s'est élargi à la société entière, se manifeste de manière accrue lors du Second conflit mondial. Elle reste pourtant en opposition avec les représentations les plus prégnantes du féminin. Pour les plus grand nombre ces femmes ne sont pas à leur place. Cette présence, au fond inacceptable, devient intolérable quand il s'agit des ennemies. Leur participation ne peut alors être que le résultat d'une immoralité intrinsèque.

Lors de la guerre de 1870, la présence de francs-tireurs, parmi lesquels quelques femmes, avait considérablement marqué et inquiété les armées allemandes. Non seulement l'existence de francs-tireurs est une infraction aux lois de la guerre, mais elle introduit une menace permanente sur l'armée régulière. Tout civil devient potentiellement un tueur. Cet imaginaire de l'ennemi invisible, du tireur qui se cache derrière chaque homme, femme ou enfant, est un des éléments de compréhension essentiel dans le déroulement des atrocités commises par l'armée du Kaiser en Belgique et au nord de la France en 1914.

Dès 1870, les Françaises étaient, aux yeux des Allemands, susceptibles de devenir un adversaire: "le curé qui pendant la guerre assassine avec l'aide de trois bonnes femmes cinq hussards prussiens dans leur sommeil se condamne lui même "(Jeismann, 1997, p.254). Bien qu'encore exceptionnel, l'engagement des femmes ennemies est perçu dans le cadre des attributs du féminin. Le courage leur est dénié. Elles tuent certes, mais lâchement, parce que 
les hommes sont endormis dans l'exemple ci-dessus, mais dans tous les cas parce qu'elles sont femmes donc a priori invisibles comme ennemi. Leur engagement n'est en aucun cas reconnu comme politique ou patriotique. Leur participation à la guerre ne peut alors être qu'attribué aux défauts des femmes: insouciance, immoralité, cupidité. C'est bien souvent pour un homme qu'elles s'engagent, qu'il soit mari, père, frère ou amant, voire curé dans l'exemple cité plus haut, comme si les ne pouvaient s'engager de leur propre volonté. En prenant les armes, c'est-à-dire, en rejetant de la manière la plus radicale leur assignation à la maternité, elles enfreignent la frontière du genre. Cette perception de l'ennemie permet de lui attribuer tous les vices. Sournoise, cruelle, dévergondée, telle est l'ennemie. Selon Yannick Ripa c'est ainsi, qu'au cours de la guerre d'Espagne, les franquistes perçoivent les miliciennes républicaines: "les rebelles déclarent la violence des républicaines contraire à la nature féminine. Elle confirme le basculement des rouges du côté de l'animalité : hyènes, fauves assoiffés de sang, les républicaines sont réputées plus cruelles plus perverses, plus sadiques que leurs compagnons de combat (...) Leur violence n'est pas politique, elle est érotique, traduction d'une sexualité débridée, stimulée par le marxisme " (Ripa, 1997, p.138).

Le discours sur la cruauté des ennemies n'est pas propre à l'imaginaire phalangiste, on retrouve des descriptions analogues en maintes occasions. C'est le cas pendant l'occupation et la libération de la France à propos des femmes agentes de la Gestapo. "Elle était bien une indicatrice des nazis. Espèce de monstre hybride, cette créature portait sans cesse des costumes d'hommes, s'exhibait en "champion" des poids et haltères, s'était même fait couper les seins pour avoir l'air plus masculin. Elle s'était mise au service de la Gestapo pour pouvoir assouvir ses instincts sadiques en torturant les patriotes (...) [Elle] a été exécutée par la résistance il y a quelques mois ". Le portrait est ici extrême. On retrouve le travestissement, ici de femme en homme, déjà évoqué comme élément de différenciation sexué de l'autreennemi. Mais celui-ci est poussé à l'extrême, les seins coupés renvoient autant mythe des Amazones, combattantes éponymes, qu'à la négation de la maternité. Elle n'est plus femme, mais hybride, violente comme un homme, cruelle par instinct, comme une femme.

Précisons que le discours qui met en scène une féminité en négatif quand il s'agit de l'ennemie a son contraire à propos des femmes de son propre camp. Cependant, qu'elles soient héroïsées ou non, leur engagement est décrit au nom des qualités féminines de dévouement, de sacrifice pour les siens. Il est également une parenthèse dans ce que doit être et demeurer une vie d'épouse, de mère.

Il n'en demeure pas moins que ces cas se situent encore dans un registre où les ennemies restent peu nombreuses, chacune plus facilement incarnée dans une sorte d'héroïne du mal. Avec la guerre d'Algérie, on se trouve dans une situation nouvelle, un grade au dessus. Les femmes de l'ennemi deviennent toutes individuellement des ennemies. L'identité de chacune, femme, Algérienne, en fait progressivement une combattante. Dans une conception militaire classique vieillards, femmes et enfants quel que soit leur camp sont de par leur faiblesse des personnes à protéger. Cette vocation protectrice du soldat, bien que très largement contredite par les faits lors des différents conflits du siècle, reste présente dans l'univers mental du combattant et de l'armée: "il est interdit de les considérer comme des ennemis". Or, le regard porté par l'armée française sur les Algériennes change au cours de la guerre ${ }^{6}$. Jusqu'à la bataille d'Alger en 1957, elles sont invisibles comme ennemi. Leur invisibilité en fait d'ailleurs aux yeux du FLN des poseuses de bombe idéales. Progressivement, leur participation à la guerre de libération transforme ces "femmes de rebelles" en "femmes rebelles". À partir de l'année 1959, il est clair pour les Français que l'ennemi s'accorde autant au féminin qu'au masculin. L'étude des journaux de marche et opérations montre bien un changement radical : “ aux X rebelles et X femmes succèdent X rebelles dont X femmes".

${ }^{5}$ L'Humanité, 20 septembre 1944.

${ }^{6}$ Ce basculement dans l'image de l'ennemi algérien est développé par Raphaëlle Branche dans le chapitre "Des victimes au féminin", BRANCHE R, La torture et l'armée pendant la guerre d'Algérie, Paris, Gallimard, 2001, p.300-309. 
Ainsi, pour la première fois l'Armée française combat un ennemi qu'elle perçoit comme mixte et doit s'adapte de fait à une situation inédite. Des sections pour femmes sont alors prévues dans les camps d'internement, des consignes rappellent qu'il faut étendre aux femmes les arrestations et interrogatoires, les exécutions sommaires comme les tortures concernent les deux sexes.

L'ennemie est une figure manifestement moins intangible que son pendant masculin. Car si la guerre est perçue comme une affaire d'hommes, la proximité croissante des femmes au front, l'extension de la guerre à la société tout entière, font que l'expérience de guerre est désormais partagée par les deux sexes. Cette présence, oblige à penser l'ennemi également comme une ennemie. Elle devient un adversaire spécifique, "un ennemi-femme", dont le corps est objet de conquête de domination. Elle demeure une image de fragilité qu'il faut masquer pour rendre possible la mise à mort de masse. Pourtant, la frontière du genre et celle qui sépare deux camps adverses se croisent plus qu'elles ne se superposent. En effet, s'il est possible de nier, d'oublier sa propre barbarie, il n'en est rien avec la moitié de son propre camp. La nécessité d'une mobilisation totale de la société, interdit d'en rejeter la moitié féminine dans le camp adverse. 